\title{
Fe Distribution in Zr-2.5Nb Pressure Tubes having Variable Deformation Properties
}

\author{
M.L. Trudeau*, A.M. Serventi* and R. Gauvin** \\ * Materials Science, Hydro-Québec Research Institute, 1800 Boul. Lionel-Boulet, Varennes, \\ Québec, Canada, J3X 1S1 \\ ** Department of Mining and Materials Engineering, McGill University, Wong Building, 3610, \\ University Street, Montréal, Québec, Canada, H3A 2B2
}

One critical component of CANDU nuclear reactor is the $\mathrm{Zr}-2.5 \mathrm{Nb}$ pressure tubes (PT). The deformation of these tubes (creep, elongation, sagging) has a major impact on the lifespan of the reactor. Even if PT materials have been extensively studied, an understanding of the deformation mechanisms and especially of the variability of this deformation from tube to tube is still not fully understood. However, recent researches seem to indicate that a large part of this variability could be explained by small variation in impurities level or some details of the fine microstructure [1].

One impurity element that has always been suspected to play a role in this variability is Fe, of which the amount in PT varies typically from about 200 to $2000 \mathrm{ppm}$ [2]. The analysis of the correlation between Fe distribution and the deformation behaviour of PT, which have shown a difference in deformation properties, is thus fundamental. Even if some studies have looked at the Fe distribution [3], the emergence of highly efficient characterization tools, coupled to Monte-Carlo simulation, can help in more systematic and detailed investigations.

In this work, Fe distribution in different $\mathrm{Zr}-2.5 \mathrm{Nb}$ PT was investigated using a HITACHI HD-2700 dedicated STEM equipped with $\mathrm{Cs}$ correction, EDS detector (Si(Li) or SDD) and EELS spectrometer. In order to minimize preparation artefacts, which have shown to be present during electropolishing [3], STEM samples were prepared using a HITACHI NB5000, nanoDUE'T focused ion beam. These tools facilitate greatly the high resolution observation of the interfaces between the $\alpha$ and $\beta$ phases, as seen in Figure-1. Moreover this combination offers high throughput elemental analysis as observed in Figure-2, which shows the presence of Fe at the interface between the $\beta$ and $\alpha$ phases. Together with Monte-Carlo simulation (Figure-2c), it is now possible to get a more complete understanding of Fe distribution in PT samples. This should help us ascertain if Fe as any impact in the variable deformation behaviour of PT materials.

Another benefit of this combination of FIB/STEM tools is the ease of the preparation of pin samples, as seen in Figure-3a, for true tomography analysis, allowing a 3D characterization of this complex structure. Furthermore, the revealing of the two phases structure, observed in the SEM micrograph of Figure-3b, was obtained directly through FIB processing $[4,5]$.

[1] M. Griffiths et al., $13^{\text {th }}$ Int. Symp. on Zr in the Nucl. Ind., ASTM STP 1423, (2002) 796.

[2] G.A. Bickel and M. Griffiths, J. Nucl. Mater. 383 (2008) 9.

[3] V. Perovic, et al., J. Nucl. Mater. 224 (1995) 93.

[4] M.L. Trudeau et al., to be published elsewhere

[5] The authors would like to acknowledge the assistance of M. Konno and J.J. Clarke from Hitachi High Technologies for some samples preparation and observation. 


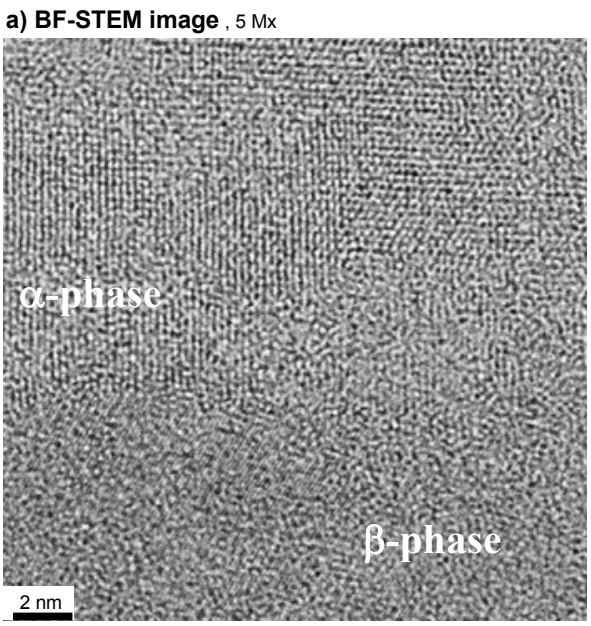

b) DF-STEM image , $5 \mathrm{Mx}$

Fig. 1 High-resolution FEG-STEM a) bright field and b) dark field images of the interface between a $\alpha$ and $\beta$ phase in a $\mathrm{Zr}-2.5 \% \mathrm{Nb}$ PT materials.
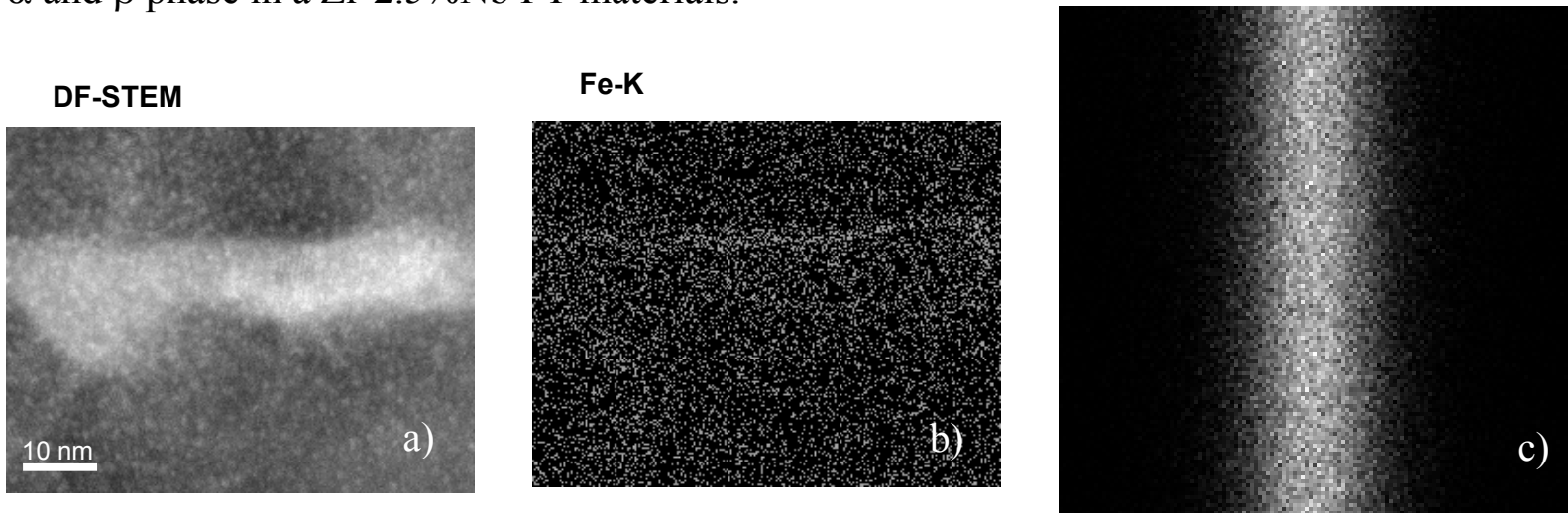

Fig. 2 a) DF-STEM image of $\alpha$ (dark) and $\beta$ (white) phases in a Zr-2.5\%Nb PT with b) rapid EDS mapping of the interface showing clearly the presence of Fe at the boundary between the $\alpha$ and $\beta$ phases and c) Monte-Carlo simulation for a $0.3 \mathrm{~nm}$ Fe layer in $\mathrm{Zr}(50 \mathrm{~nm}$ thick sample at $200 \mathrm{kV})$.
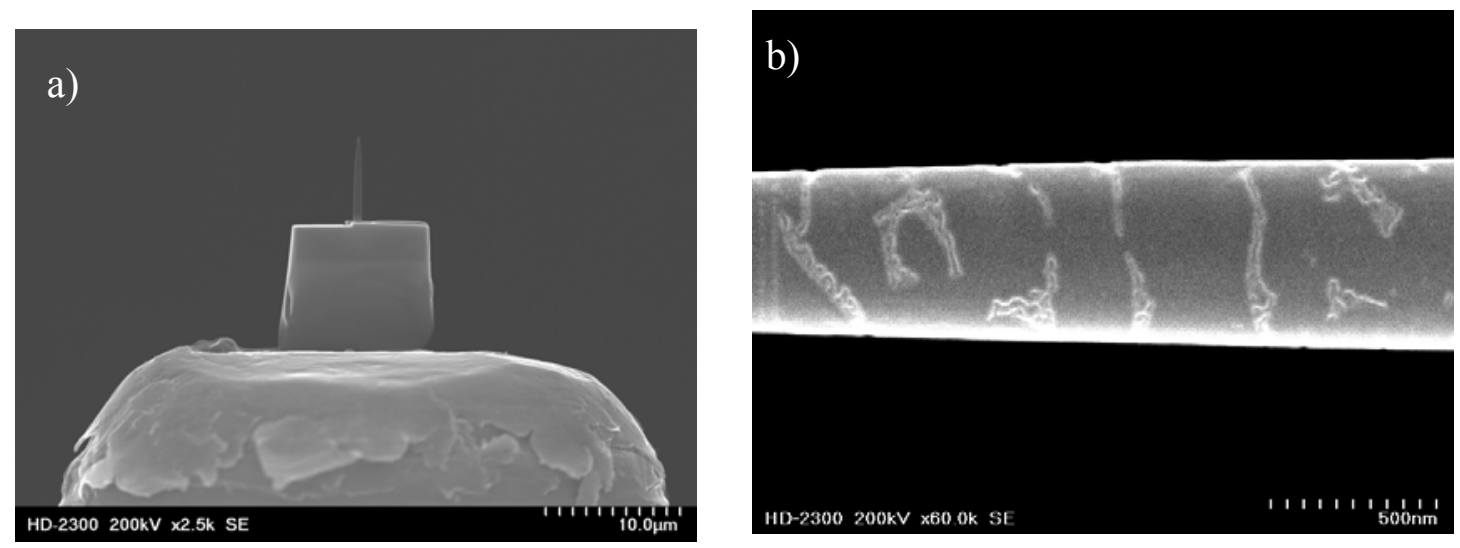

Fig.3 SEM micrograph of a FIB tomography $\mathrm{Zr}-2.5 \mathrm{Nb}$ sample a) at low magnification and b) at higher magnification revealing easily the complex microstructure of this sample (these two micrographs were obtained on a HD-2300 dedicated STEM at $200 \mathrm{kV}$ ). 